\title{
DIGITALCOMMONS
}

5-1-2009

\section{Bias in Stabilized Sieve Sampling}

\author{
Liming Guan \\ University of Hawaii at Manoa, lguan@hawaii.edu \\ John P. Wendell \\ University of Hawaii at Manoa, john.wendell@hawaii.edu
}

Follow this and additional works at: http://digitalcommons.wayne.edu/jmasm

Part of the Applied Statistics Commons, Social and Behavioral Sciences Commons, and the Statistical Theory Commons

\section{Recommended Citation}

Guan, Liming and Wendell, John P. (2009) "Bias in Stabilized Sieve Sampling," Journal of Modern Applied Statistical Methods: Vol. 8 : Iss. 1 , Article 23.

DOI: $10.22237 /$ jmasm/1241137320

Available at: http://digitalcommons.wayne.edu/jmasm/vol8/iss1/23

This Regular Article is brought to you for free and open access by the Open Access Journals at DigitalCommons@WayneState. It has been accepted for inclusion in Journal of Modern Applied Statistical Methods by an authorized editor of DigitalCommons@WayneState. 


\section{Bias in Stabilized Sieve Sampling

\author{
Liming Guan John P. Wendell \\ University of Hawaii at Manoa
}

The stabilized sieve sample selection method (SSM) is considered to be a probability proportional to size (PPS) sampling method with an unbiased estimator (Horgan 1997, 1998). This article demonstrates that SSM does not select items with PPS and that the point estimator is biased.

Key words: Sampling with probability proportional to size; Hansen-Hurwitz estimator; HorvitzThompson estimator.

\section{Introduction}

Consider a situation where it is desired to make an inference about an unknown population parameter, $Y$, such that

$$
Y=\sum_{I=1}^{N} y_{I}
$$

where $N$ is the population size, $I=(1,2, \ldots N)$, and $y_{I}$ is unknown but can be determined exactly by applying some procedure. An unbiased estimate of $Y$ can be obtained when sampling with replacement using the Hansen-Hurwitz estimator (Brewer \& Hanif 1983, p. 5)

$$
\hat{Y}_{H H}=\frac{1}{n} \sum_{i}^{n} \frac{y_{i}}{p_{i}}
$$

where $n$ is the sample size, $y_{i}$ is the value $y_{I}$ that is determined for the $i$ th item in the sample, $p_{I}$ is the probability of inclusion as the $i$ th item in the sample of the population item $I$ and $p_{i}$ is the value of $p_{I}$ for the $i$ th item selected for the sample. Note that under sampling with replacement an individual population item, $I$, can be included in the sample more than once.

Liming Guan is an Associate Professor of Accounting in the Shidler College of Business. Email:1guan@hawaii.edu. John P. Wendell is a Professor of Accounting in the Shidler College of Business. Email: john.wendell@hawaii.edu.
When sampling without replacement, an unbiased estimate of $Y$ can be obtained using the Horvitz-Thompson estimator (Brewer \& Hanif 1983, p. 6)

$$
\hat{Y}_{H T}=\sum_{i=1}^{n} \frac{y_{i}}{\pi_{i}}
$$

where $\pi_{I}$ is the probability of inclusion in the sample of the population item $I$ and $y_{i}$ is the value of $y_{I}$ for the $i$ th item in the sample.

Equations (2) and (3) are general and allow for an unbiased estimate of $Y$ regardless of how $p_{I}$ or $\pi_{I}$ are determined. For sampling with equal probabilities $p_{I}=1 / N$ and $\pi_{I}=n / N$. Sampling with unequal probabilities is often a good choice and may be the only possible method given the sampling frame. Examples of sampling with unequal probabilities are stratified sampling and cluster sampling. Another method for sampling with unequal probabilities is probability proportional to size (PPS) sampling. The size variable can be any variable $x$ for which every $x_{I}$ satisfies

$$
0<x_{I}<\frac{X}{n} \text { where } X=\sum_{I=1}^{N} x_{I} .
$$

The right side of the inequality is a requirement only when sampling without replacement. If these conditions are met then a PPS sample can be drawn by setting

$$
p_{I}=\frac{x_{I}}{X}
$$

when sampling with replacement and 


\section{GUAN \& WENDELL}

$$
\pi_{I}=n \frac{x_{I}}{X}
$$

when sampling without replacement.

PPS sampling methods are generally applicable to any population where it is desired to estimate $Y$ using either (2) or (3) and there is a size variable available conforming to (4). This article examines the properties of two such methods, the sieve method and the stabilized sieve method (SSM).

\section{Sieve Sampling}

The sieve method is a PPS sampling without replacement method that was developed by Rietveld (1978, 1979a,b). The presentation of the method given here is based on Horgan (1998). A population item is selected for inclusion in the sample if it satisfies the inequality

$$
r_{I} \leq x_{I}
$$

where $r_{I}$ is a random variable uniformly distributed on the interval $(0, X / n)$ and each $r_{I}$ is independently generated. It is important to note that the realized sample size, $n_{r}$, is a random variable that will not always be the same as $n$. Equation (3) with the sum over $n_{r}$ and $\pi_{I}$ defined as in (6) will yield an unbiased estimate of $Y$. The properties of the sieve method and the SSM will be illustrated by sampling from a hypothetical population with $N=5$ and $n=2$ used by Wright (1991) to demonstrate that systematic PPS samples lose their PPS property when augmented by systematically sampling the remaining population. The details of this population are given in Table 1 .

Table 1: Test Population. $N=5, n=2, X=20$.

\begin{tabular}{|c|c|c|c|c|}
\hline$I$ & $x_{I}$ & $\pi_{I}$ & $1-\pi_{I}$ & $p_{I}$ \\
\hline 1 & 2 & 0.2 & 0.8 & 0.10 \\
\hline 2 & 3 & 0.3 & 0.7 & 0.15 \\
\hline 3 & 4 & 0.4 & 0.6 & 0.20 \\
\hline 4 & 5 & 0.5 & 0.5 & 0.25 \\
\hline 5 & 6 & 0.6 & 0.4 & 0.30 \\
\hline
\end{tabular}

Table 2: Probabilities of sample outcomes for the test population in Table 1 for sieve sampling. Column $j$ is an identification variable for each of the 32 outcomes. The second column indicates which population items were included in a particular sample outcome and $p$

\begin{tabular}{|c|c|c|}
\hline$j$ & Is & $p$ \\
\hline 1 & Null & 0.0672 \\
\hline 2 & 1 & 0.0168 \\
\hline 3 & 2 & 0.0288 \\
\hline 4 & 3 & 0.0448 \\
\hline 5 & 4 & 0.0672 \\
\hline 6 & 5 & 0.1008 \\
\hline 7 & 1,2 & 0.0072 \\
\hline 8 & 1,3 & 0.0112 \\
\hline 9 & 1,4 & 0.0168 \\
\hline 10 & 1,5 & 0.0252 \\
\hline 11 & 2,3 & 0.0192 \\
\hline 12 & 2,4 & 0.0288 \\
\hline 13 & 2,5 & 0.0432 \\
\hline 14 & 3,4 & 0.0448 \\
\hline 15 & 3,5 & 0.0672 \\
\hline 16 & 4,5 & 0.1008 \\
\hline 17 & $1,2,3$ & 0.0048 \\
\hline 18 & $1,2,4$ & 0.0072 \\
\hline 19 & $1,2,5$ & 0.0108 \\
\hline 20 & $1,3,4$ & 0.0112 \\
\hline 21 & $1,3,5$ & 0.0168 \\
\hline 22 & $1,4,5$ & 0.0252 \\
\hline 23 & $2,3,4$ & 0.0192 \\
\hline 24 & $2,3,5$ & 0.0288 \\
\hline 25 & $2,4,5$ & 0.0432 \\
\hline 26 & $3,4,5$ & 0.0672 \\
\hline 27 & $1,2,3,4$ & 0.0048 \\
\hline 28 & $1,2,3,5$ & 0.0072 \\
\hline 29 & $1,2,4,5$ & 0.0108 \\
\hline 30 & $1,3,4,5$ & 0.0168 \\
\hline 31 & $2,3,4,5$ & 0.0288 \\
\hline 32 & $1,2,3,4,5$ & 0.0072 \\
\hline
\end{tabular}
is the probability of that outcome. 


\section{BIAS IN STABILIZED SIEVE SAMPLING}

These sample outcome probabilities are calculated as

$$
p_{j}=\prod_{I \in s_{j}} \pi_{I} \prod_{I \notin s_{j}} 1-\pi_{I}
$$

where $s_{j}$ is the jth sample outcome in Table 2 . For example, the probability of getting sample outcome 11 , item 2 and 3 , is $0.3 \times 0.4 \times 0.8 \times$ $0.5 \times 0.4=0.0192$. That the sieve method is indeed PPS for this population can be checked by summing the probabilities for each sample outcome containing a particular population item, $I$, and verifying that it is equal to the value for $\pi_{I}$ in Table 1 .

Table 3 shows the probability of achieving a particular $n_{r}$. These probabilities can be calculated from Table 2 by summing all probabilities for outcomes of a given size.

Table 3: Probabilities of a realized sample size for the test population in Table 1 .

\begin{tabular}{|c|c|}
\hline$n_{r}$ & $p$ \\
\hline 0 & 0.0672 \\
\hline 1 & 0.2584 \\
\hline 2 & 0.3644 \\
\hline 3 & 0.2344 \\
\hline 4 & 0.0684 \\
\hline 5 & 0.0072 \\
\hline
\end{tabular}

Table 4 shows the probabilities of inclusion in $n_{r}$ for each combination of population item and realized sample size for the test population in Table 1. These conditional probabilities are not proportional to $x_{I}$.

Table 4:. Conditional probabilities of inclusion.

\begin{tabular}{|c|c|c|c|c|c|c|}
\cline { 2 - 7 } \multicolumn{1}{c|}{} & \multicolumn{6}{c|}{$n_{r}$} \\
\hline$I$ & 0 & 1 & 2 & 3 & 4 & 5 \\
\hline 1 & 0 & 0.0650 & 0.1658 & 0.3242 & 0.5789 & 1 \\
\hline 2 & 0 & 0.1115 & 0.2700 & 0.4863 & 0.7544 & 1 \\
\hline 3 & 0 & 0.1734 & 0.3908 & 0.6314 & 0.8421 & 1 \\
\hline 4 & 0 & 0.2601 & 0.5247 & 0.7389 & 0.8947 & 1 \\
\hline 5 & 0 & 0.3901 & 0.6487 & 0.8191 & 0.9298 & 1 \\
\hline
\end{tabular}

The stabilized sieve method (SSM) (Horgan 1997, 1998) is a modification of the sieve method that ensures that the final sample size is always equal to $n$. This section details how the method selects items for a sample and then considers the properties of point estimators of $Y$ for samples selected using the SSM.

The SSM is selected in two stages. First an initial sample, $S_{l}$, is selected using (7). In the second stage the sampling process is conditioned upon the number of items in $S_{l}$ (Horgan 1998)

$$
S_{2}=\left\{\begin{array}{ccc}
S_{1}+\mathrm{A}\left(n-n_{r}\right) & \text { if } & n_{r}<n \\
S_{1} & \text { if } & n_{r}=n \\
S_{1}-\mathrm{R}\left(n_{r}-n\right) & \text { if } & n_{r}>n
\end{array}\right.
$$

where $S_{2}$ is the final sample and $\mathrm{A}(m)$ and $\mathrm{R}(m)$ are defined as follows: $\mathrm{A}(m)$ selects $m$ items one at a time (with replacement) by taking a simple random sample of one from the entire population (including items in $S_{l}$ ) and this item is selected for inclusion in the sample if

$$
r \leq x_{I}
$$

where $r$ is a uniformly distributed random number in the interval $\left(0, \max \left(x_{I}\right)\right]$. The process is repeated, generating a new value for $r$ each time, until $m$ items are selected. All items selected using $\mathrm{A}(m)$ satisfy $(5)$. $\mathrm{R}(m)$ selects $m$ items to remove from $S_{1}$ by taking a simple random sample of size $m$ from $S_{l}$.

Table 5 gives the probabilities for each sample outcome and $n_{r}$ for the population in Table 1 sampled using SSM. Because of the complexity of (9) some explanation of how individual cells in this table were calculated may be useful. The simplest case is when $n_{r}=2$ where the values are taken directly from Table 2 . Outcome $9(2,5)$ with $n_{r}=4$ will be used to illustrate the cases when $n_{r}>2$. First, the probabilities of all the outcomes where $n_{r}=4$ and both item 2 and 5 are present (outcomes 28, 29 , and 31) are summed and then divided by the number of combinations of two items that can be drawn from a population of four items. This gives $(0.0072+0.0108+0.0288) / 6=0.0078$. When $n_{r}<2$, there may be more than one path to a sample outcome. For example, outcome $7(2,3)$ 


\section{GUAN \& WENDELL}

with $n_{r}=1$ can occur when the initial sieve sample contains only item 2 or only item 3 . Outcome 3 in Table 2 gives the probability of $S_{l}$ containing only item 2 and Table 1 gives the value of $p_{I}$ for selecting item 3 in the second stage. The probabilities that $S_{1}$ contains only item 3 and that item 2 is selected in the second stage can be determined in the same manner. Thus, the probability for outcome 7 when $n_{r}=1$ is $0.0288 \times 0.20+0.0448 \times 0.15=0.01248$.

Table 5 shows probabilities for sample outcomes for the test population in Table 1 for stabilized sieve sampling. Column $j$ is an identification variable for each of the 15 outcomes. The second column indicates which population items were included in a particular sample outcome, $n_{r}$ is the realized sample size in stage one and the cells contain the joint probability of the sample outcome and $n_{r}$. Table 6 provides $p_{I}$ for the population in Table 1 when sampling with SSM and demonstrates that these probabilities are not PPS. These probabilities are derived from Table 5 by summing the probabilities for each sample outcome that contains a particular $I$ divided by $n$, which is 2 in this case. For outcomes where $I$ is included twice it is counted twice.

Horgan (1998, equations 8, 17, and 19) provides an estimator for $Y$ that is conditional upon $n_{r}$ :

$$
\hat{Y}_{s}=w X \sum_{i=1}^{n} \frac{y_{i}}{x_{i}} \text { where } w=\left\{\begin{array}{ccc}
\frac{1}{2 n-n_{r}} & \text { if } \quad n_{r}<n \\
\frac{1}{n} & \text { if } \quad n_{r}=n \\
\frac{n_{r}}{n^{2}} & \text { if } \quad \mathrm{n}_{\mathrm{r}}>n
\end{array}\right.
$$

Although (11) conditions on $n_{r}$, it does not take into consideration that the probabilities of inclusion in the sample given $n_{r}$ are not proportional to $x_{I}$ (see Table 4). Consequently, $\hat{Y}_{s}$ is a biased estimator. The expected value of $\hat{Y}_{s}$ for the test population can be calculated by determining $\hat{Y}_{s}$ for every cell in Table 5, multiplying the result by the probability in the cell and then taking the sum of those products. The result is $1.0917 y_{1}+1.0877 y_{2}+1.0824 y_{3}+$ $1.0749 y_{4}+1.0637 y_{5}$.
Because the SSM method is a sampling with replacement method based on the sieve method, and the sieve method is a PPS method without replacement it seems reasonable to use $\hat{Y}_{H H}$ with $p_{I}$ calculated according to (5). This will also give a biased estimate of $Y$, the expected value of which is $0.9491 y_{1}+0.9693 y_{2}+1.0029$ $y_{3}+1.0111 y_{4}+0.9791 y_{5}$ for the Table 1 population.

It is possible to construct an unbiased estimator of $Y$ when using the SSM with the population in Table 1. This is done by first setting each $p_{I}$ to the corresponding value in Table 6 and then calculating $\hat{Y}_{H H}$ accordingly. Unfortunately, the use of this estimator is limited to very small populations because it requires an enumeration of all $2^{N}$ possible sample outcomes for the stage 1 sieve sample.

\section{Conclusion}

The stabilized sieve method does not sample with PPS and that both $\hat{Y}_{S}$ and $\hat{Y}_{H H}$ with $p_{I}$ calculated according to (5) are biased estimators of $Y$. Further, the calculation of the unbiased estimator is prohibitively expensive to compute for any but the smallest populations. Nonetheless, the SSM performed well in the simulations in Horgan (1997 and 1998) in comparison to the sieve method and the probability proportionate to size with replacement method (PPR).

All three of these methods have drawbacks, either the possibility of items showing up more than once in the sample (SSM, PPR) or variable sample size (sieve), or bias (SSM). Systematic PPS sampling methods utilizing a random sort of the population before application have none of these drawbacks because they select fixed size samples without replacement with probabilities that are exactly proportional to $x_{I}$ (see Brewer \& Hanif 1983, procedures 2 and 3 ). These selection methods are easily applied with modern computers if both $I$ and $x_{I}$ are available in a computer accessible file. Consequently, with these sampling frames the systematic procedures should be preferred over either the sieve, SSM, or PPR methods. However, not all sampling frames make the entire population $x_{I}$ conveniently accessible by computer and the sieve, SSM, and PPR methods 


\section{BIAS IN STABILIZED SIEVE SAMPLING}

may have some practical advantages with these sampling frames that offset their disadvantages. With such challenging sampling frames, the SSM method should not be ruled out simply because of the difficulty in achieving a completely unbiased estimate of $Y$, particularly if the population characteristics and sample sizes are similar to those used for the simulations in Horgan (1997 and 1998).

\section{References}

Brewer, K. R. W., \& Hanif, M. (1983). Sampling with unequal probabilities. NY: Springer-Verlag.

Horgan, J. M. (1997). Stabilizing the sieve sample using PPS. Auditing: A Journal of Practice and Theory, 16, 40-51.
Horgan, J. M. (1998). Stabilized sieve sampling: A point-estimator analysis. Journal of Business and Economic Statistics, 16, 42-51.

Rietveld, C. (1978). De Zeefmethode Als Selectiermethode Voor Statistiche Steekproeven in de Controlepaktijk (I). Compact: Computer en Accountant, 15, 2-11.

Rietveld, C. (1979a). De Zeefmethode Als Selectiermethode Voor Statistiche Steekproeven in de Controlepaktijk (II) en (III). Compact: Computer en Accountant, 16, 2-13.

Rietveld, C. (1979b). De Zeefmethode Als Selectiermethode Voor Statistiche Steekproeven in de Controlepaktijk (IV). Compact: Computer en Accountant, 17, 9-18.

Wright, D. W. (1991). Augmenting a sample selected with probability proportional to size. Auditing: A Journal of Practice and Theory, 10, 145-158.

Table 5: Probabilities of sample outcomes for the test population in Table 1 for stabilized sieve sampling.

\begin{tabular}{|c|c|c|c|c|c|c|c|}
\cline { 3 - 8 } \multicolumn{1}{l|}{} & \multicolumn{6}{c|}{$n_{r}$} \\
\hline$j$ & $I \mathrm{~s}$ & 0 & 1 & 2 & 3 & 4 & 5 \\
\hline 1 & 1,1 & 0.00067 & 0.00168 & 0 & 0 & 0 & 0 \\
\hline 2 & 1,2 & 0.00202 & 0.00540 & 0.00720 & 0.00760 & 0.00380 & 0.00072 \\
\hline 3 & 1,3 & 0.00269 & 0.00784 & 0.01120 & 0.01093 & 0.00480 & 0.00072 \\
\hline 4 & 1,4 & 0.00336 & 0.01092 & 0.01680 & 0.01453 & 0.00540 & 0.00072 \\
\hline 5 & 1,5 & 0.00403 & 0.01512 & 0.02520 & 0.01760 & 0.00580 & 0.00072 \\
\hline 6 & 2,2 & 0.00151 & 0.00432 & 0 & 0 & 0 & 0 \\
\hline 7 & 2,3 & 0.00403 & 0.01248 & 0.01920 & 0.01760 & 0.00680 & 0.00072 \\
\hline 8 & 2,4 & 0.00504 & 0.01728 & 0.02880 & 0.02320 & 0.00740 & 0.00072 \\
\hline 9 & 2,5 & 0.00605 & 0.02376 & 0.04320 & 0.02760 & 0.00780 & 0.00072 \\
\hline 10 & 3,3 & 0.00269 & 0.00896 & 0 & 0 & 0 & 0 \\
\hline 11 & 3,4 & 0.00672 & 0.02464 & 0.04480 & 0.03253 & 0.00840 & 0.00072 \\
\hline 12 & 3,5 & 0.00806 & 0.03360 & 0.06720 & 0.03760 & 0.00880 & 0.00072 \\
\hline 13 & 4,4 & 0.00420 & 0.01680 & 0 & 0 & 0 & 0 \\
\hline 14 & 4,5 & 0.01008 & 0.04536 & 0.10080 & 0.04520 & 0.00940 & 0.00072 \\
\hline 15 & 5,5 & 0.00605 & 0.03024 & 0 & 0 & 0 & 0 \\
\hline
\end{tabular}

Table 6. Probabilities of inclusion in a sample draw for each item in the test population in Table 1 compared to the probability under PPS.

\begin{tabular}{|c|c|c|}
\hline \multirow{2}{*}{$I$} & \multicolumn{2}{|c|}{$p_{I}$} \\
\cline { 2 - 3 } & actual & PPS \\
\hline 1 & 0.09491 & 0.10 \\
\hline 2 & 0.14540 & 0.15 \\
\hline 3 & 0.19805 & 0.20 \\
\hline 4 & 0.25277 & 0.25 \\
\hline 5 & 0.30886 & 0.30 \\
\hline
\end{tabular}

